\title{
Current Developments in Time-resolved X-ray Diffraction
}

\author{
Simone Techert \\ Department Structural Dynamics of (Bio)chemical Systems \\ Max-Planck-Institute for Biophysical Chemistry \\ Am Fassberg 11
}

37077 Göttingen

\section{Introduction}

For all processes in nature, in which chemistry is involved, reformations of functional groups, bond breaking and / or bond formation processes are essential. This is as true for small molecular systems in interstellar clouds as for complex biological functionalities in living cells or organisms. Since the invention of quantum physics at the beginning of last century it is possible to describe the properties of small molecules in the gas phase in a more or less exact way. With increasing number of atoms and increasing complexity, however, the mathematical precision of this theoretical description decreases and structure-function relationships might no longer be evident or predictable.

By precisely measuring the time-evolution of structural changes with pumped x-ray sources, and correlating the measured entities with optical properties, it is possible to derive structure-function-relationships in a time-dependent manner or as a function of time [1-25]. The time-dependence of these relationships and the development of relevant methodologies is current scientific interest in our workgroup. Systems of investigation are organic materials with optical functionalities like dyes [3,4,7,8,9,13], sensor systems, opto-electronical materials [10] or chromophores $[8,9,19,20]$ on the one hand, and enzymes on the other hand. In the following, special interest will be given to organic materials in the solid state. 


\section{Experimental Technique: Optical Light Pump / X-ray Probe Scheme}

During the last 20 years, a long-lasting progress has been made in the field of timeresolved x-ray diffraction (TR-XRD) and photo-crystallography, where the structure factors of a system are measured as a function of time and irradiation. Laser systems have been developed rapidly, and the combination of pulsed laser sources with pulsed x-ray sources, particularly by using synchrotron x-ray radiation and x-rays generated by plasma sources, has made the application of pump-probe schemes routine (as it has been in optical spectroscopy during the last decades).

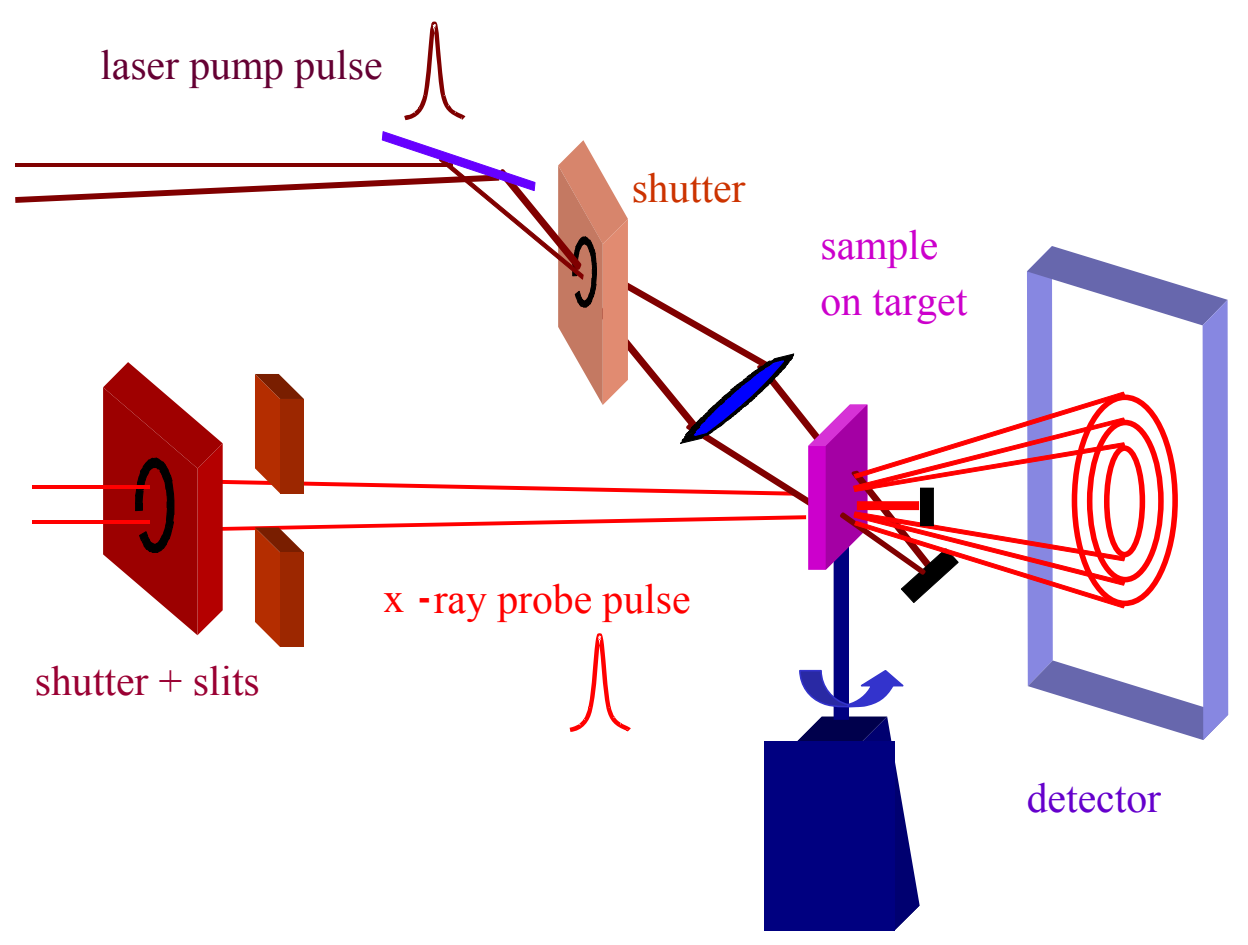

Figure 1: Principle of the optical light pump / x-ray probe set-up in time-resolved x-ray diffraction.

The experimental set-up of such a time-resolved experiment is summarised in Figure 1. After photoinitiation of the reaction by an optical laser pulse, the excited state is probed with an x-ray pulse. By varying the delay between pump and probe, a series of snapshots of the moving structure monitored by the change of the $\mathrm{x}$-ray diffraction pattern is taken. Note, that 
each shot probes the average structure (non-excited and excited, including the dispersion of the latter) at a given time $t_{i}$. The advantage of such a pump-probe experiment, over temperature jump, pressure jump or concentration jump experiments, is the well-defined time zero with respect to the system investigated, here the molecule. Using photo-absorption as the reactioninitiating step, it is additionally possible to gain highest time-resolution during the experiment and to guide the reaction into one particular direction or into one particular reaction pathway.

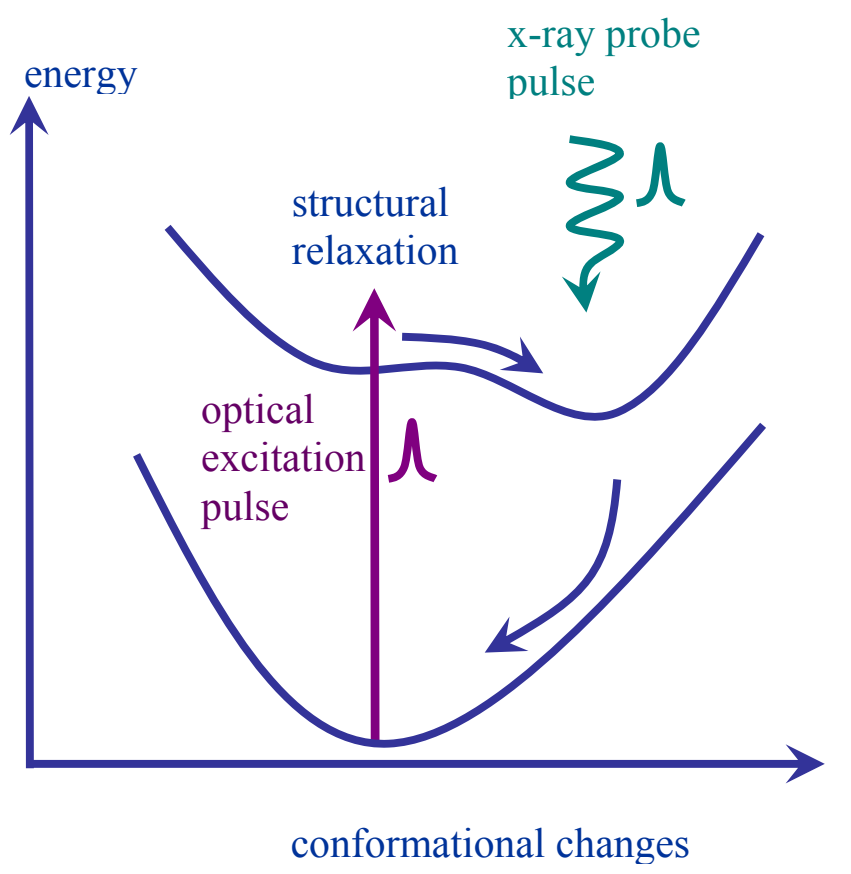

Figure 2: Scheme of an optical light pump / x-ray probe experiment. After photoexcitation of a reaction, a cascade of structural relaxation and reorganisation processes is initiated which can be probed by an $\mathrm{x}$-ray pulse.

Figure 2 summarises an example of which molecular effect can be investigated by time-resolved x-ray diffraction. The (ultra)fast pump light initiates the reaction in the NIR to UV range. After this photoinitiation, a cascade of structural relaxation and reorganisation processes follows which can be probed by an x-ray pulse applying various diffraction [1-13] or X-ray absorption $[14,15]$ techniques.

The application of pump probe schemes not only allows the determination of quasistationary intermediate structures but also the investigation of the time course of a reaction, by 
measuring the intensity changes of a diffraction pattern as a function of time $[3,4,13]$. Thus, it is possible to apply both the classical tools of x-ray crystallography and x-ray diffraction developed during the past decades and, additionally, the mathematics developed for kinetic experiments. In a kinetic description of a system, the systematic time dependences on the concentration decrease of the reactant states and the concentration increase of the product states are studied. This approach is valid even for fast time scales like picoseconds and shortliving intermediates of a reaction as long as the thermal equilibrium of the microenvironment is guaranteed.

In an analogous way to electrochemistry or spectroscopy kinetic experiments, time-resolved xray diffraction experiments can employ time-dependent kinetic "monitoring tools". In this case, one or more Bragg diffraction peaks can be followed as function of time by comparing complex diffraction patterns (such as Laue diffraction patterns) [6].

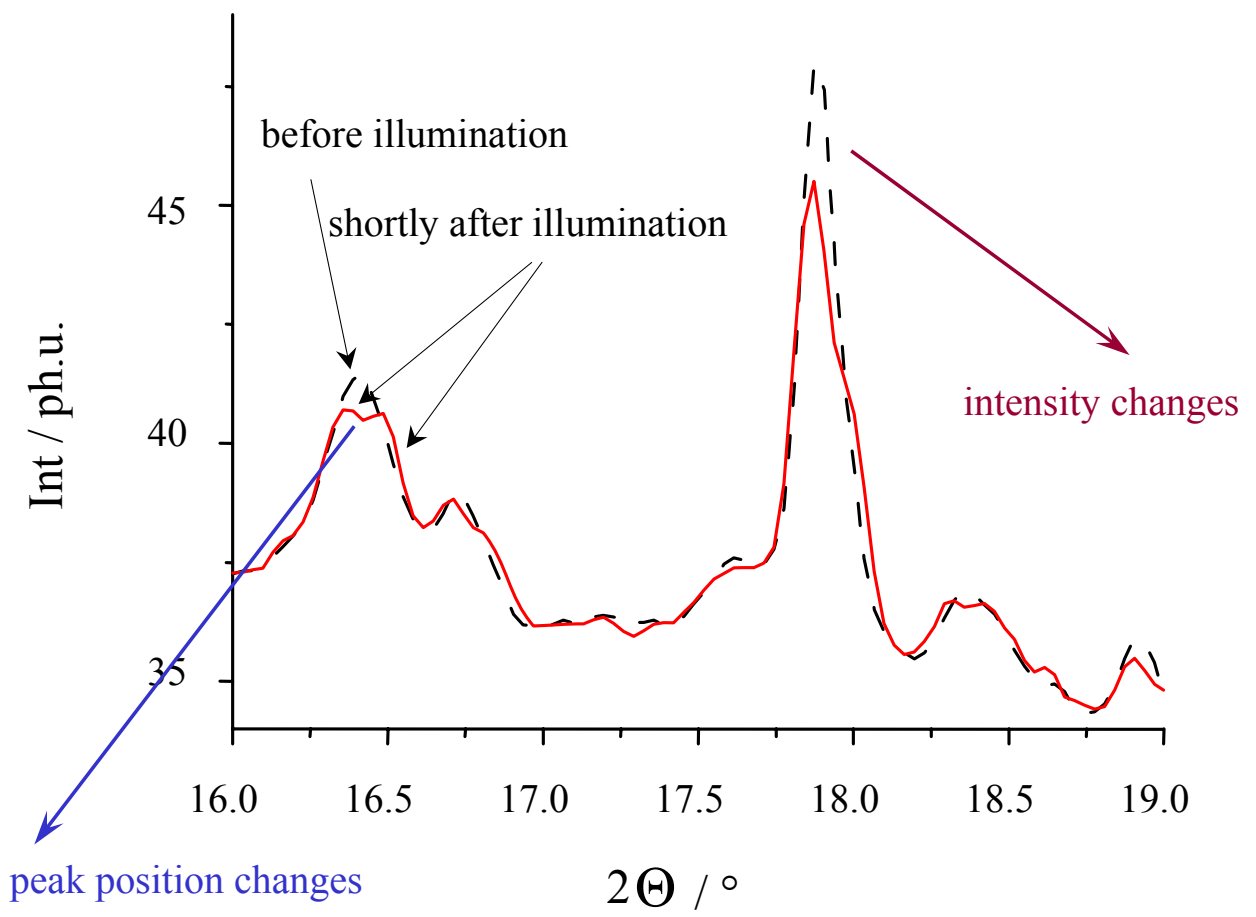

Figure 3: Possible effects of light-illumination on a powder diffraction pattern.

Figure 3 summarises the possible effects on a time-resolved powder diffraction pattern with (solid line) and without (dashed line) illumination. The peak position changes are related to the 
translational properties of the lattice, i.e. the variation of the lattice constants and unit cell dimensions. The unit cell dimensions increase or decrease upon heating or cooling or pressure changes. In light-induced experiments, in general, laser illumination leads to a warming-up of the sample and an increase of the volume, which might be investigated by the decrease of the Bragg diffraction peak positions (if the effect is strong). On the microscopic level, the propagation of acoustic modes is responsible for these modulations. Since acoustic modes propagate with speed of sound through the lattice, the time scale, where position changes occur, is strongly dependent on the grain size in the powder [7].

On the other hand, light excitation also induces intensity variations of the Bragg reflection peaks. The intensities of the peaks correlate via the square of the structure factor with the geometry of a molecule. Since geometrical changes extend the whole time scale - they can occur on the femtosecond time scale and can last up to seconds - the intensity modulations take also place on the femtosecond up to second time scale.

Figure 4 summarises the time scales of molecular effects which are possible in the solid and condensed phase [7]. In one approach of optical light pump / $\mathrm{x}$-ray probe experiments, the slow time scales of crystal reaction to light can stroboscopically be covered. Here, the time delay between laser-pump and x-ray probe is fixed so that quasi-stationary structures of transient species can be investigated. With this method mainly microsecond long-living intermediates of reversible light-driven processes are characterised. For the diffraction mode, these experiments were realised applying $\mathrm{x}$-ray home sources and synchrotron radiation $[1,5]$. The method is very powerful for measuring accurate structures of intermediates up to the microsecond time scale. On the second to millisecond time scale, thermodynamical transformations, transport reaction as well as spin-relaxed phenomena like photo-induced ferromagnetism or ferroelectricity can be studied (Figure 4) [10,22]. 
The nanosecond to picosecond time scale is characterised through the electronic lifetimes of electronically excited singlet states and some slow relaxation processes which quench the optical response of material, or light excitation (like fluorescence or phosphorescence) [16,17]. These relaxation processes are rotational motions or vibrational motions like librations, large amplitude motions or acoustic modes. Vibrational motions pf higher frequencies dominate the femtosecond time scale.

$\begin{array}{llllll}\mathrm{s} & \mathrm{ms} & \mathrm{HS} & \mathrm{ns} & \mathrm{ps} & \mathrm{fs}\end{array}$

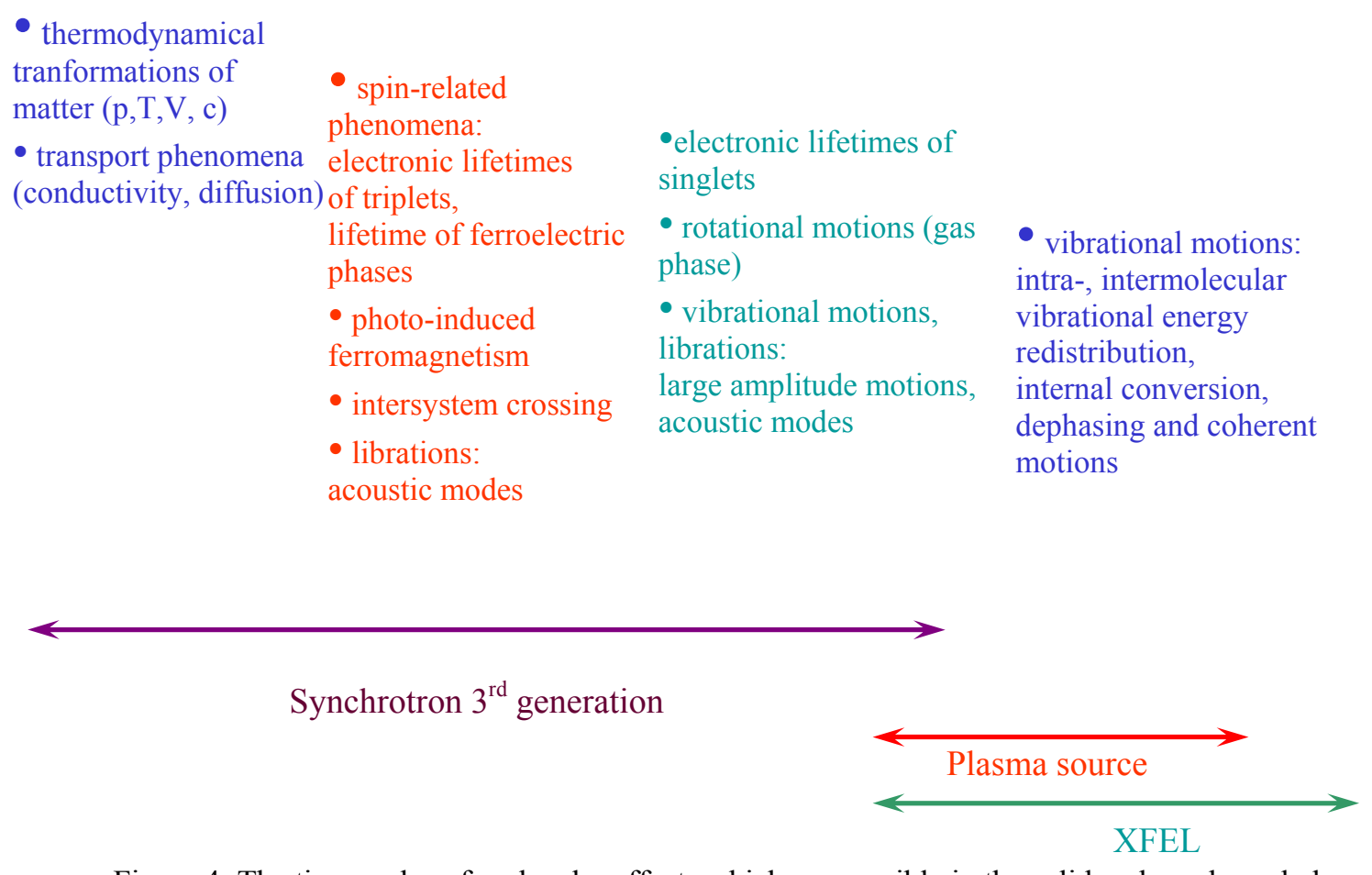

Figure 4: The time scales of molecular effects which are possible in the solid and condensed phase.

To use optical light excitation as reaction initiation has the practical advantage that the light penetrates the reaction vessel with a velocity which is proportional to the speed of light times the refraction index of the material. Therefore one photon immediately initiates a reaction, if it is absorbed by a chromophore. Rapid diffusion-controlled equilibrium processes, as they occur in temperature or pressure jump experiments, are the reaction steps which determine the kinetics of the system on a longer time scale.

However, there exist two concrete problems when investigating photo-induced processes: 
According to the Woodward-Hofman rules and depending on the symmetry of the system, photochemical and thermally initiated reaction products are exclusively formed. As a consequence of this rule, photochemically allowed reactions are thermally forbidden and vice versa. This can be explained by the fact that the photon has a spin of one and, er spin- and energy conservation the product states must be different compared to heat jump experiments. However, this general limitation resulting in the fact that photo-induced processes might not always be simulated by temperature-initiated processes, is valid for the whole photochemical field independent of the probe source used (infrared, Raman, x-ray, etc.).

The second, more general problem concerns particularly pump/probe schemes, where optical light is used as pump source and X-rays are used as probe source (or vice versa) and is caused by the fact that the cross sections of the scattered $\mathrm{x}$-ray photons $\left(\mathrm{ca} .10^{-15} \mathrm{~cm}^{2}\right)$ are two orders of magnitude smaller than the cross sections of the optical photons $\left(\mathrm{ca} .10^{-13} \mathrm{~cm}^{2}\right)$ [7]. Figure 5 summarises these circumstances: in general, the optical density oD of a molecular system is defined as the product of molar extinction coefficient $\varepsilon$, absorption length $d$ and concentration $C$ of the absorbing species. Especially the latter is very high in chromophoreic crystals or solutions with high concentrations of dyes $\left(\mathrm{c} \approx 10^{-2} \mathrm{M}\right)$, and when using organic molecules containing phenyl moieties (with extinction coefficients of about $\varepsilon=20000 \mathrm{~mol} / \mathrm{cm}$ ), the optical density of the material can easily reach a value of $o D=4-5$ for a crystal with $d=100$ $\mu \mathrm{m}$ thickness in the main absorption band or a solution of the dye in a capillary with $100 \mu \mathrm{m}$ thickness. Applying the Lambert-Beer law (with $I=I_{o} \exp [-\varepsilon c d]$ ) optical photons just penetrate only some micrometers into the crystal yielding a small non-homogeneous excitation located mainly at the surface of the crystal. Figure 5a, left hand side (dotted line) shows such low penetration of optical light due to the high chromophore concentration in the crystal and a high extinction coefficient. In contrast (Figure $5 \mathrm{~b}$, dotted line), reasonable $\mathrm{x}$-ray probe wavelengths of $\lambda_{\mathrm{x} \text {-ray }}=1-2 \AA$ penetrate more than $100 \mu \mathrm{m}$ into the crystal due to the low $\mathrm{x}$-ray 
cross section. According to Figure 5, the optimal geometric match between optical light pump and $\mathrm{x}$-ray probe would be reached with $\mathrm{x}$-ray wavelengths of $\lambda_{\mathrm{x} \text {-ray }}=7-10 \AA$ which are represented as dashed lines in Figure 5a (right) and Figure 5b (left). Figure 5b (inset i1) shows a simulation of a powder diffraction pattern for the $\mathrm{Si} \mathrm{K}_{\alpha}$ line at $\lambda_{\mathrm{x} \text {-ray }}=7.1 \AA$. The small amount of reflections is not enough for a statistical relevance guiding to a satisfying structure refinement of a transient species with unknown geometry.

a)

b)

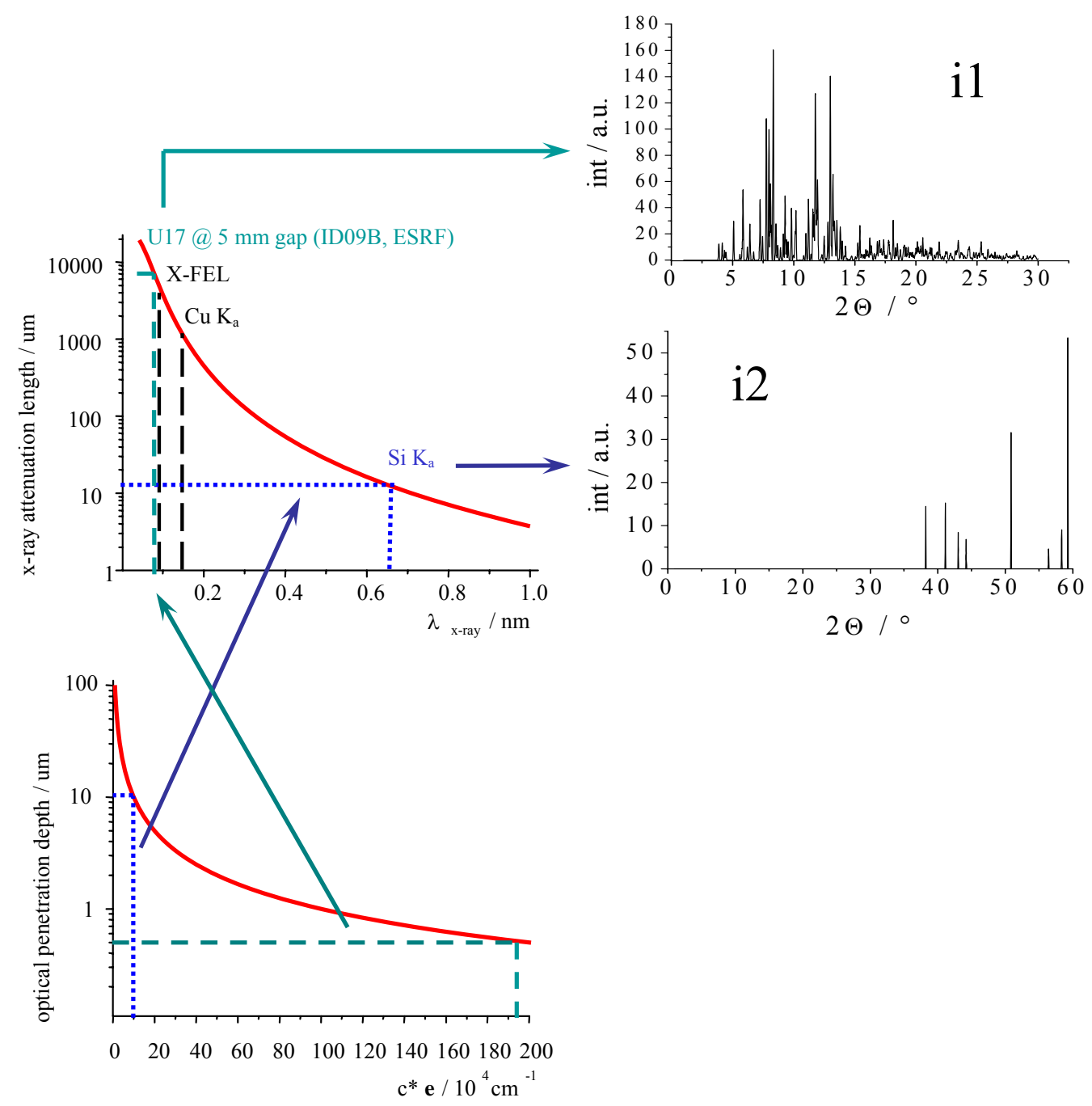

Figure 5: Difference in penetration depth for optical light pulses and $\mathrm{x}$-ray probe pulses. The problem of penetration depth rises for every chromophoric system in the condensed or solid state when a critical amount of concentration of the chromophore is reached. 
On the other hand, monochromatic light selected from an in-vacuum undulator, like the U17 at the ID09 beamline of the ESRF in Grenoble, allows the collection of a powder diffraction pattern up to very high resolution in space (Figure 5 b inset i2). Due to the long penetration depth of the monochromatic x-ray radiation $\left(\lambda_{\mathrm{x}-\mathrm{ray}}=0.738 \AA\right)$ of some hundred micrometers and in contrast to the optical penetration depth of some micrometers, for systems with high optical extinction coefficients, it is optimal to work in the case of powdereous materials with grain sizes smaller than $<1 \mu \mathrm{m}$ rather than with single crystals in the micrometer range - which brings us to powder diffraction studies. Here, a homogeneous optical excitation is guaranteed, which can be probed by any x-ray wavelength allowing also small wavelengths leading to high spatial resolution $[3,4,13]$. Other possibilities of a homogeneous excitation of a single crystal might also be to use the optical "trick" of cooperative excitations and investigating the light-induced structural changes by oscillation diffractometry as it will be also discussed in the following [10].

\section{Correlation Functions and Kinetic Description}

In classical kinetcis the quantitative description of the time courses are given by correlation functions which we would like to recall:

For a general reaction like

$$
\alpha A+\beta B+\gamma C \longrightarrow \text { products, }
$$

where $\alpha, \beta$ and $\gamma$ are the stoichiometric factors and $[A],[B]$ and $[C]$ are the concentrations of the reactants, the time law is given by

$$
-\frac{1}{\alpha} \frac{d[A]}{d t}=k_{n}[A]^{p}[B]^{q}[C]^{r} \text {. }
$$

$p, q$ and $r$ are the order of the reaction. Analogously, the time laws for $\mathrm{B}$ and $\mathrm{C}$ are defined as 


$$
-\frac{1}{\beta} \frac{d[B]}{d t}=k_{n}[A]^{p}[B]^{q}[C]^{r} \text { and }-\frac{1}{\gamma} \frac{d[C]}{d t}=k_{n}[A]^{p}[B]^{q}[C]^{r} \text {. }
$$

The total order of the reaction is $n=p+q+r$.

For a reaction of first, second and third order, the time dependences are well known [29] and in terms of the correlation function described as

first order: $k t_{1}=\ln \left(\frac{1}{C_{n}(t)}\right)$,

second order: $k t_{2}=\frac{1}{b}\left[\frac{1}{C_{n}(t)}-1\right]$,

third order: $k t_{3}=\frac{1}{2 b^{2}}\left[\frac{1}{C_{n}(t)}-1\right]$

with $b$ being determined by the concentration of the reactants [4].

In cases of a linear dependence of the concentration of reactants and products to the intensity of the Bragg diffraction peak, i.e. in a non-kinematic description of the reactant-product concentrations in the light-transformed sample, the correlation function $C_{n}(t)$ is defined as

$$
C_{n}(t)=\frac{I(\Theta, t)-I(\Theta, t=\infty)}{I(\Theta, t=0)-I(\Theta, t=\infty)},
$$

where $I(\Theta, t)$ is the intensity of a Bragg diffraction peak at scattering angle $\Theta$ and time $t$, the reaction start is at $t=0$ and the end of the reaction is at $t=\infty$. Note, that in this description the population of the light-transformed species is proportional to the structure factor of the light-induced structure $\left|F_{h k l}^{\text {light }}(t)\right|^{2}$ as the same applies for the non-transformed part of the sample, respectively.

If the photo-induced effects create local photo-excited centers and not the whole probed crystal volume is transformed, a correlation function based on common crystallographic evaluation schemes can be derived as: 
$C_{n}(t)=\frac{{ }^{1} N^{\text {light }}(t) F_{h k}^{\text {light }}(t)-{ }^{1} N^{\text {light }}(t=\infty) F_{h k}^{\text {light }}(t=\infty)}{{ }^{1} N^{\text {light }}(t=0) F_{h k l}^{\text {light }}(t=0)-{ }^{1} N^{\text {light }}(t=\infty) F_{h k l}^{\text {light }}(t=\infty)}$

where ${ }^{1} N^{\text {light }}(t)$ is the occupancy of the light-induced molecules in the crystal lattice and $F_{h k l}^{\text {light }}(t)$ its structure factor of this species as a function of time. As in common kinetic experiments, the order of reaction can now be determined by plotting the correlation functions $C_{n}(t)$ (eqs. 4 and 5) against $t$. The order of reaction can therefore be distinguished by a logarithmic, linear ( $A k)$ or quadratic dependence $\left(A^{2} k\right)$ of the correlation function from $t$.

\section{Investigations on Short-living Intermediates of Organic Chromophores in the Crystalline State and Photo-induced Solid State Reactions}

In our TR-XRD experiments the optical excitation conditions were typically chosen in such a way, that mainly the electronically first excited state $S_{1}$ is populated, which is responsible for the colour of the organic solids. The typical electronic lifetime of this state is in the nanosecond range [7]. Picosceond TR-XRD is an appropriate tool for the investigation of the equilibrium geometry in the excited states after structural relaxation on the pico- and femtosecond time domain. In the following we will summarise the results of structural changes of molecular crystals on one side and on one-dimensional organic conductors on the other side. The overview will be finished by a short representation of our recent results on the determination of mechanistical aspects of homogeneous and heterogeneous solid state photodimerisation reactions. 


\section{1 $N, N$-Dimethylaminobenzonitrile (DMABN) in the Solid State}

In reference [3] we investigated the geometry of the short-living electronically first excited state of solid $N, N$-dimethylaminobenzonitrile $(\mathrm{DMABN})$. The relaxation process and population of this short living state in crystallline $N, N$-dimethylaminobenzonitrile can be summarised as follows (Figure 6a): After absorption of UV light (wavelength $267 \mathrm{~nm}$, puls duration $300 \mathrm{fs}$ ), the statistically light-excited chromophores are in a state that can be structurally characterised by the twist of the methyl amino groups out of the ring plane of the benzene moiety $\left(\Phi_{\text {tors }}=9.5^{\circ}\right)$ and a planar surrounding of the amino nitrogen atom $\left(\vartheta_{\text {inv }}=3^{\circ}\right)$ in the excited state (ground state structure: $\Phi_{\text {tors }}=0^{\circ}$ and $\vartheta_{\text {inv }}=7^{\circ}[32]$ ).
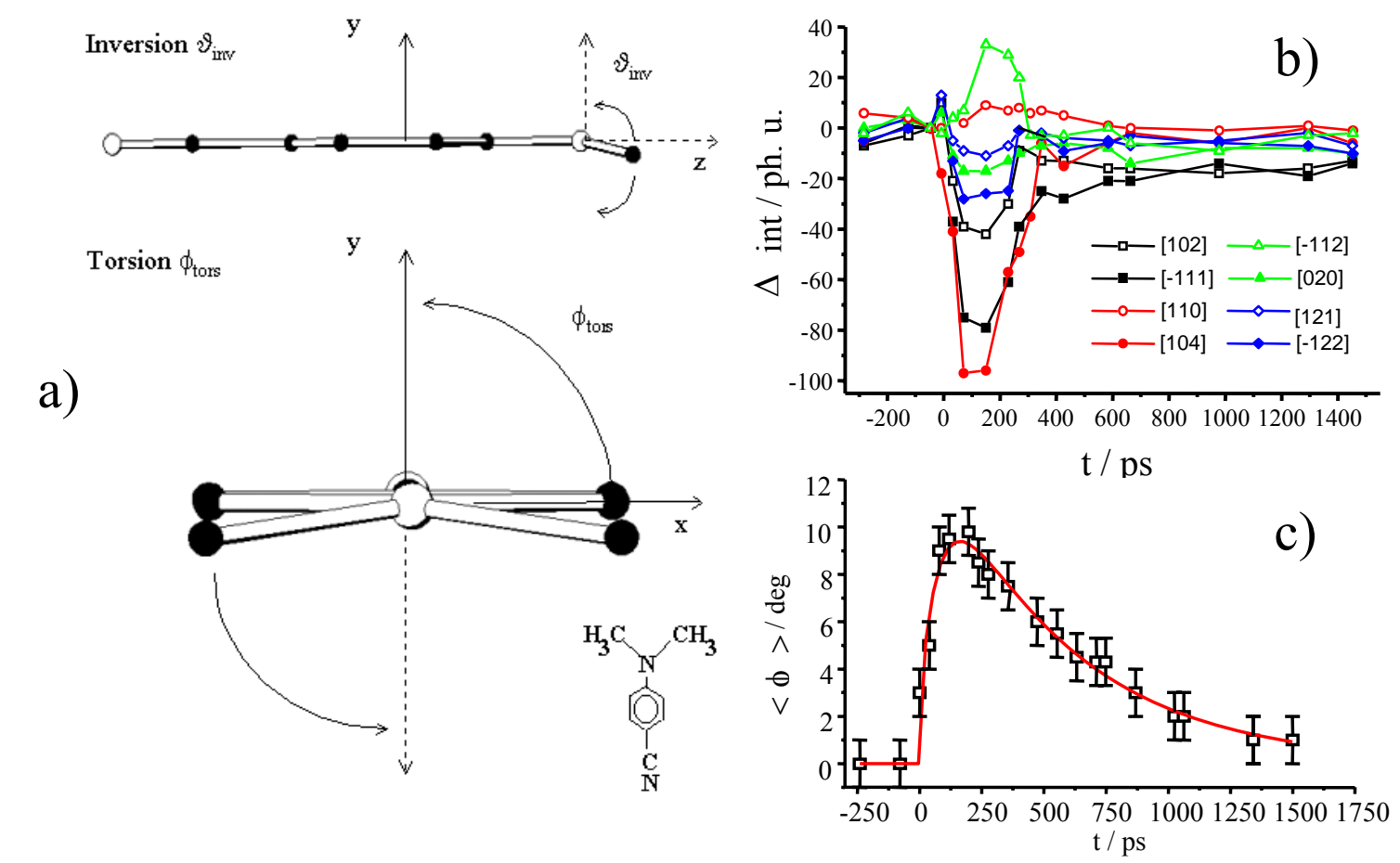

Figure 6: a) Refined conformational changes in crystalline DMABN after photoexcitation: inversion and torsion of the supposed electron donor moiety $N, N$-dimethylamino- with respect to the supposed electron acceptor moiety benzonitrile. b) Relative intensity changes of the various Bragg peaks as a function of time. The intensity changes are assigned according to the Miller indices of the corresponding reflections. c) Occupancy lifetime of the structural intermediate of DMABN.

This geometry is similar to the torsional angle found in the low-temperature structure of DMABN $\left(11^{\circ}\right)$. The structure was refined according to the procedure described in reference 
[3]. The positive and negative intensity changes of the Bragg diffraction peaks, shown in Figure $6 b$, indicate that the refined structure is not a result of laser heating. The intensity changes are signed according to the Miller indices of the corresponding reflections. The occupancy lifetime of the intermediate structure (Figure 6c), represented as the averaged tosional angle $<\Phi>$, which is the occupancy times the structural life time, could be refined to about $0.6 \mathrm{~ns}( \pm 0.4 \mathrm{~ns})$. This time is somewhat shorter than the fluorescence lifetime of the chromophore in a single crystal (between 1.5-2 ns, depending on the pureness of the material). Therefore, we think that interactions between the occupied electronic and (intermolecular) vibrational states might also play some role as driving forces for the population of the structural intermediate found.

\subsection{N,N-Diisopropylaminobenzonitrile (DIABN) in the Solid State}

TR-XRD was used in order to study the structural dynamics of $N, N$-diisopropylaminobenzonitrile [13]. The excitation conditions were similar to those of DMABN. Spectroscopic results suggest that - unlike to DMABN [3] - crystalline DIABN shows molecular charge-transfer character, i.e. a charge delocalisation in the excited state [13]. This state is populated directly after photo-excitation with an electronic lifetime of $4 \mathrm{~ns}$.

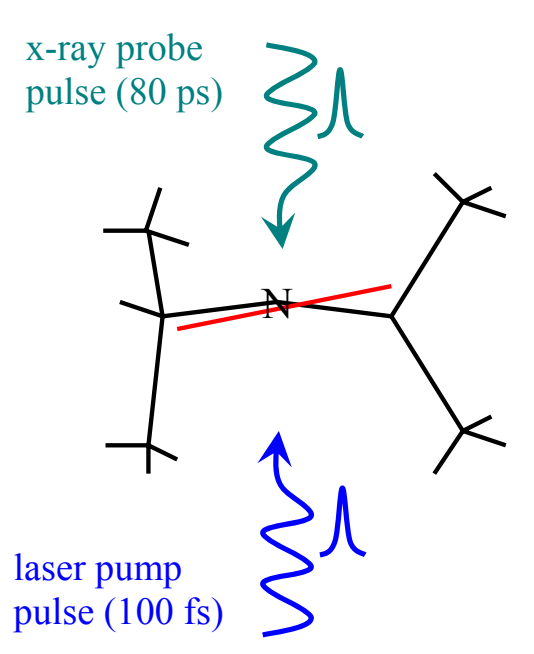

electronic ground state: $\Phi=14^{\circ}$

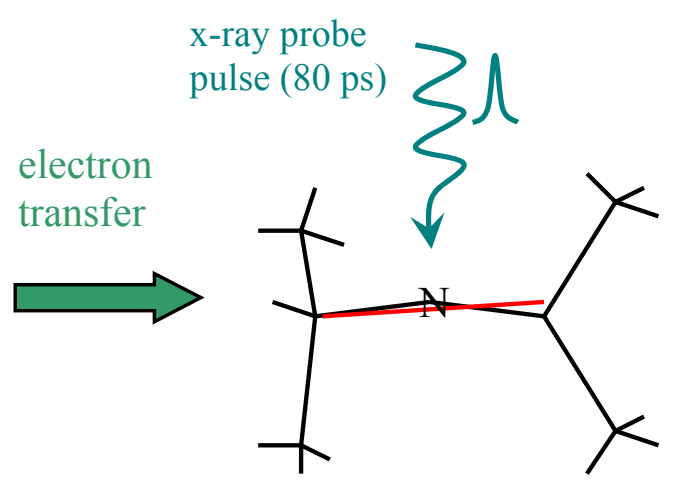

intramolecular charge transfer state: $\Phi=10^{\circ}$ 
Figure 7: Refined amino twist angle $\Phi$ of the diisopropylamino group of crystalline DIABN in a view along the long axis of the molecule before and after photoexcitation and creation of the intramolecular charge transfer state.

Interestingly, for DIABN a complementary structural rearrangement compared to the ones of DMABN in the excited state was found. The amino groups - partially out-of-plane rotated in the electronic ground state $\left(\Phi_{\text {tors }}=14^{\circ}\right)$ - reorganise to the plane of the ring $\left(\Phi_{\text {tors }}=\right.$ $10^{\circ}$ ) for the equilibrated charge transfer state. The occupancy lifetime of this structural intermediate was fitted to ca. $6.3 \mathrm{~ns}( \pm 2.3 \mathrm{~ns})$. Therefore we conclude that the refined intermediate structure belongs to the charge-separated state.
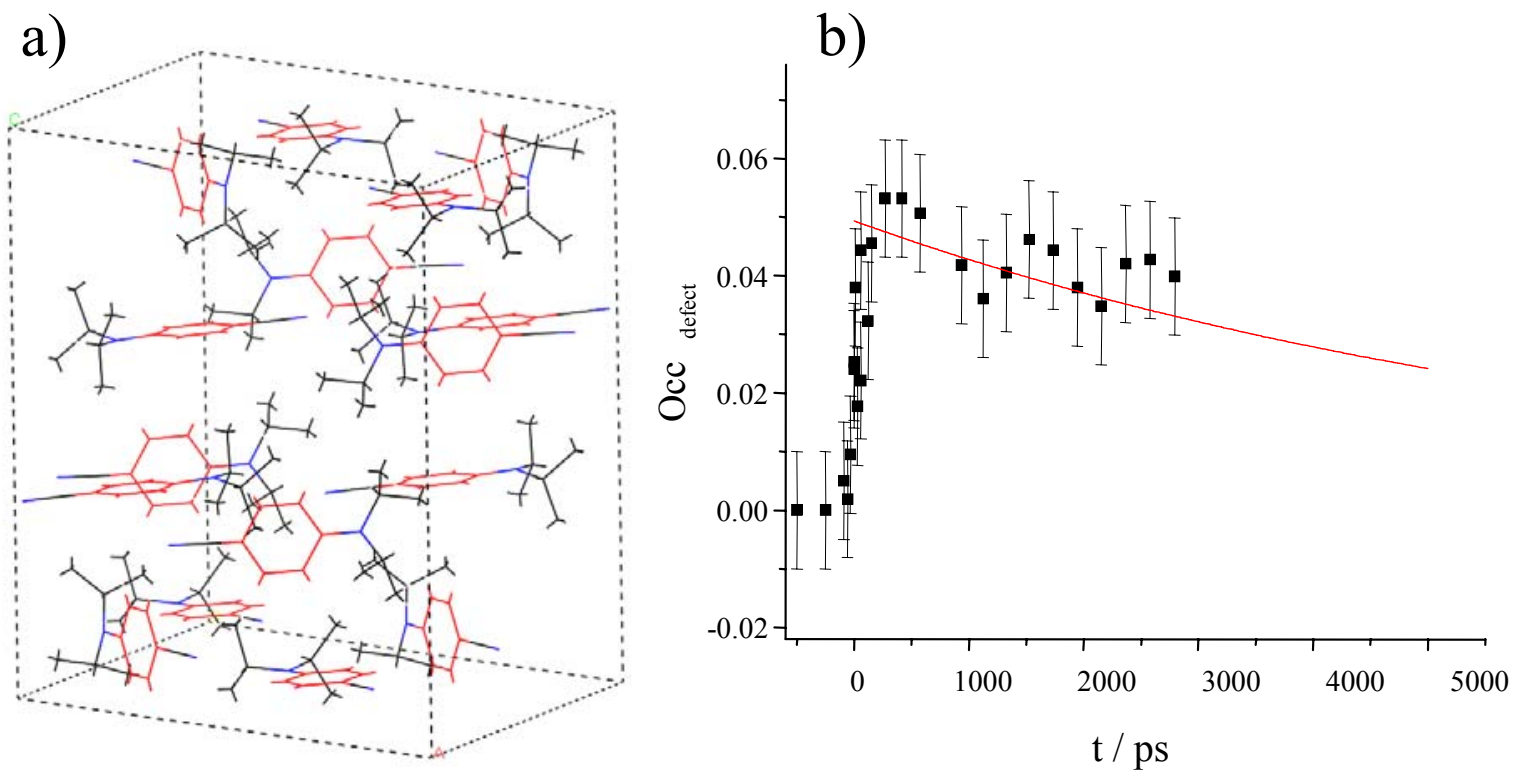

Figure 8: a) The unit cell of DIABN in a presentation, in which all molecules are in the excited state. The atoms are given in CPK colors. b) Time course of the occupancy of the photo-induced defect- structure of DIABN.

In conclusion we think that one of the reasons for the different behaviour in the structural responses upon photoexcitation of DMABN versus DIABN is certainly the different extent of electronic coupling between the electron donor and the electron acceptor which is determined by the redox potentials of the electron donor and electron acceptor energies (properties of the optical spectra). Furthermore, the sterical flexibility of the electron donor in 
the crystal lattice (DMA compared to DIA), which leads to variations of the stacking of the chromophores in the crystal lattice (and to the difference in space group symmetry), determines the structural response of the system after photoexcitation (topological effects in molecular crystal systems). Electron-phonon as well as phonon-phonon interactions of the intermolecular and intramolecular modes also contribute to the relaxation pathways of the systems in the solid state. Due to the complexity of this field, further experimental investigations have been started.

\subsection{The One Dimensional Organic Conductor Tetrathiafulvalene Chloranil (TTF-CA)}

In DMABN and DIABN the photo-excited "defect centers" are statistically distributed over the crystal lattice and the electron phonon coupling mainly occurs localised in such a way, that phonons, belonging to intramolecular large amplitude motions, couple with intermolecular phonons (like librations). In the system presented in the following, tetrathiafulvalene-chloranile (TTF-CA), a strong electron-phonon coupling occurs between the electron donor center tetrathiafulvalene and the electron acceptor center chloranile and the intermolecular modes which propagate through the lattice. This coupling facilitates intermolecular structural rearrangements so that under special thermodynamic conditions and photo-induced long-range ordering can be achieved ( as photo-induced phase transformation [18]).

In TTF-CA the electron donor and electron acceptor are two different molecules building up in alternating stacks in the crystal lattice (in contrast to DIABN i.e., where the electron donor and electron acceptor can be found in one molecular unit) (Figure 9). Therefore the structural reorganisation, which follows the photo-induced electron transfer between TTF and CA has to propagate through the lattice as ,strings“"- leading to complete domain formation (Figure 9, left) $[10,18]$ - providing that the temperature of the system is properly tuned close to the phase transition temperature. In TTF-CA, the two phases of interest are neutral (paraelectric) and ionic (ferro-electric) and can be optically distinguished by a colour change of the 
crystal (from yellow to red) and crystallographically by a change of the space group of the system (Figure 9, right). The latter results in an increase and decrease of Bragg diffraction peaks according to the change in space group symmetry (in the ionic phase, reflections with $h k l$ with $k=2 n+1$ are strongly allowed, wheareas in the neutral phase they are forbbiden). The structural response of TTF-CA near the phase-transition temperature was studied with TRXRD. Because of the well-defined crystallography of the system, time-resolved oscillation diffractometry (a stroboscopic method) was employed.

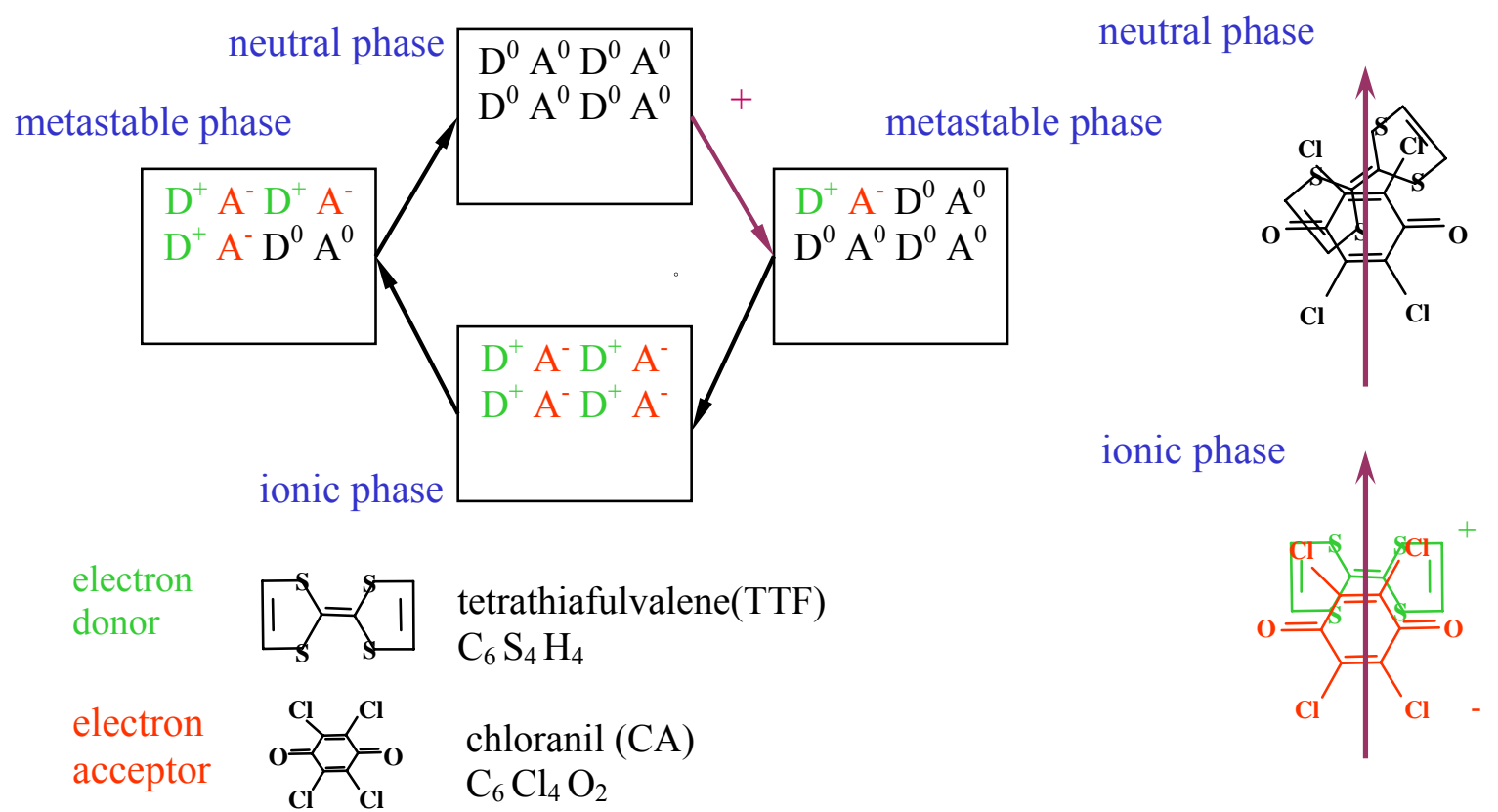

Figure 9: Left: Top: Mechanism of the photo-induced phase transition in the charge transfer system TTFCA. Bottom: the structure of the molecular units. Right: Schematic drawing of the symmetry breaking during the phase transition (view along stacking axis, symbolised as arrow) [10].

Due to the stroboscopic character of the measurements, it was possible to refine the structure of the photo-excited phase, which was a mixture of neutral and ionic phase. It was found that the photo-induced structural phase-transformation occurs within some hundred picoseconds and that the transient phase formed by that last up to some microseconds. Figure 10 shows the time evolution of the structural changes during the phase transition on some representative Bragg diffraction peaks which are marked according to their Miller indices. 


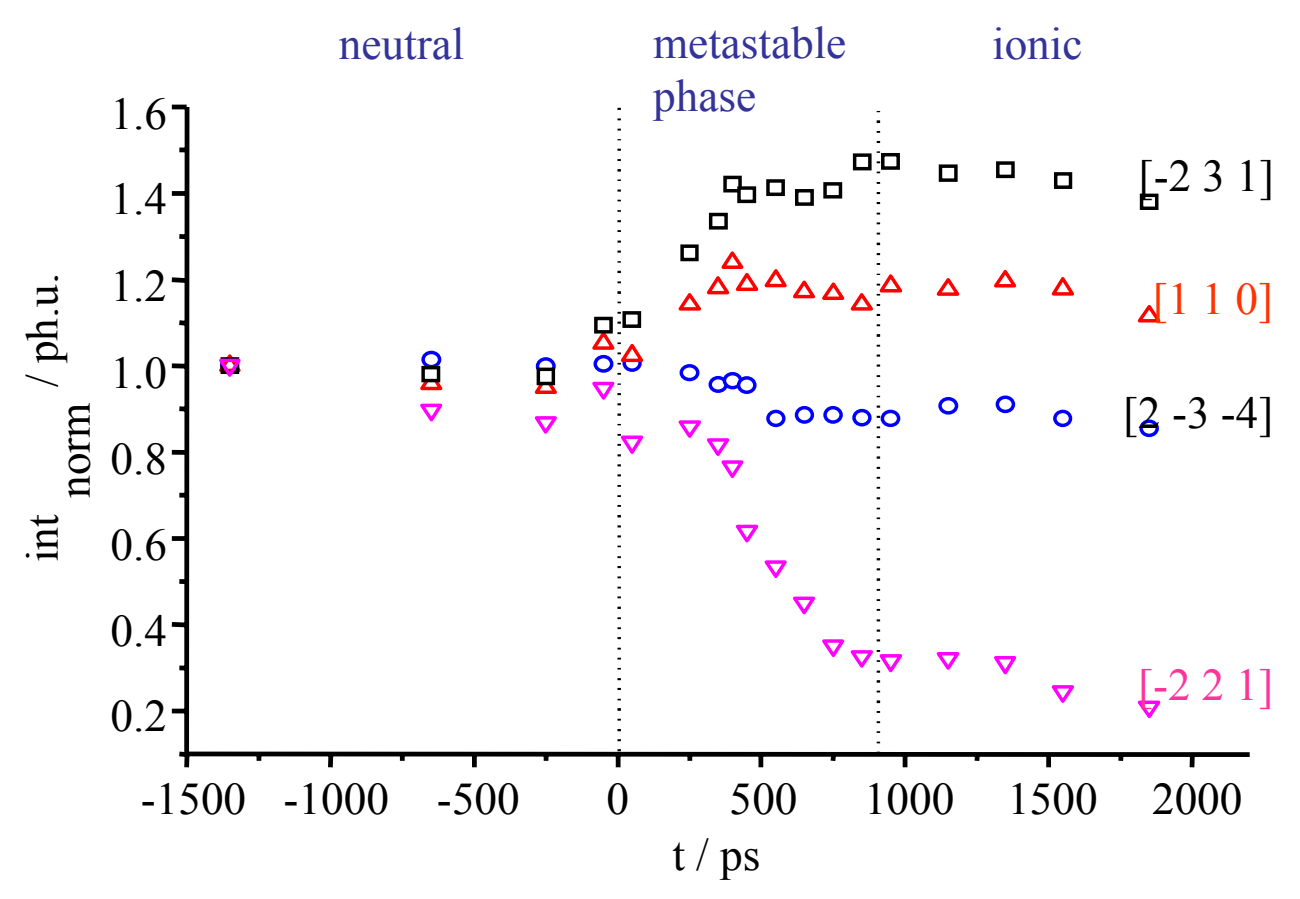

Figure 10: The time-evolution of the structural changes during the photo-induced phase transition shown on some representative Bragg diffraction peaks which are marked according to their Miller indices.

\subsection{Photocrystallographic Studies of Heterogeneous Photodimerisation Reactions in the}

\section{Solid State}

One of the basic bimolecular reactions in the solid state is the so-called topochemical reaction. The topochemical principle was first mentioned in 1931 [23] and since that time, a large school has emerged that studies this class of reaction. According to the topochemical principle, $[2+2]$ photodimerisations take place in the crystal, if the intermolecular distances between two reaction partners is smaller than $r=4.2 \AA$ (Figure 11). Crystal modifications with intermolecular distances $r>4.2 \AA$ are not photoactive. Further morphological differences emerge from head-tail ( $\alpha$-type) and head-head ( $\beta$-type) arrangements of the chromophores in the crystal. With the dimerisation of $\alpha$-type crystals leading to centrosymmetric products, and the dimerisation of $\beta$-type crytals resulting in mirror-symmetric products (Figure 11), the reaction is highly stereoselective. 


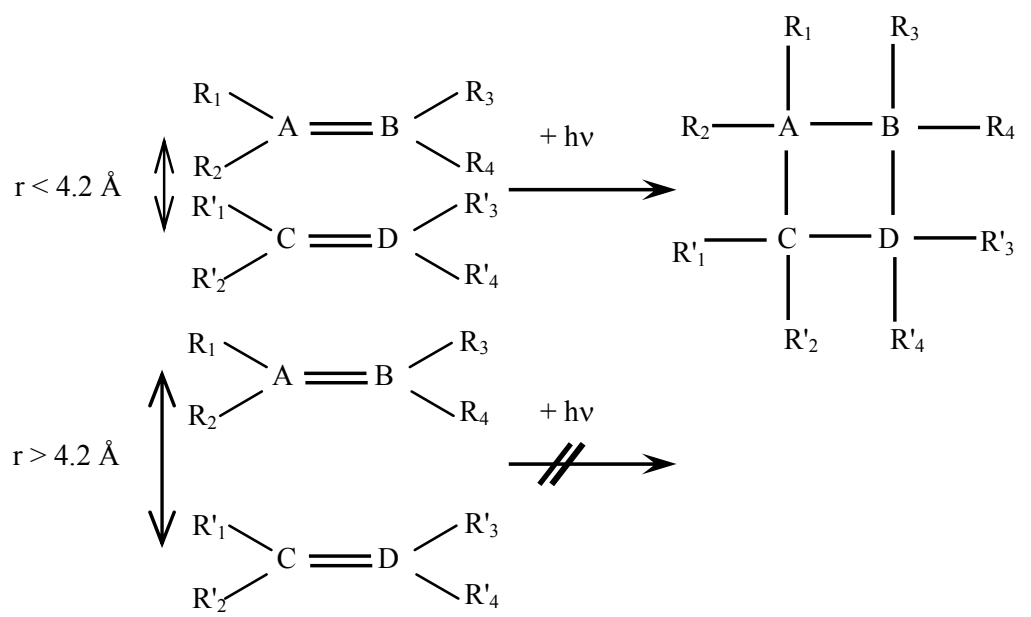

Figure 11: Symmetry criteria of the topochemical principle.

Alternatively, the topochemical principle can also be interpreted as follows: The chromophores are surrounded by a free volume that is defined by the distances between the chromophores and its left and right neighbours [24-26]. Only reactions with small geometrical changes for which the activation volume of the transition state is smaller than the free volume of reactant and product are topochemically allowed. Reactions that proceed via a transition state with enlarged activation volume are disfavoured for sterical reasons (Figure 12).

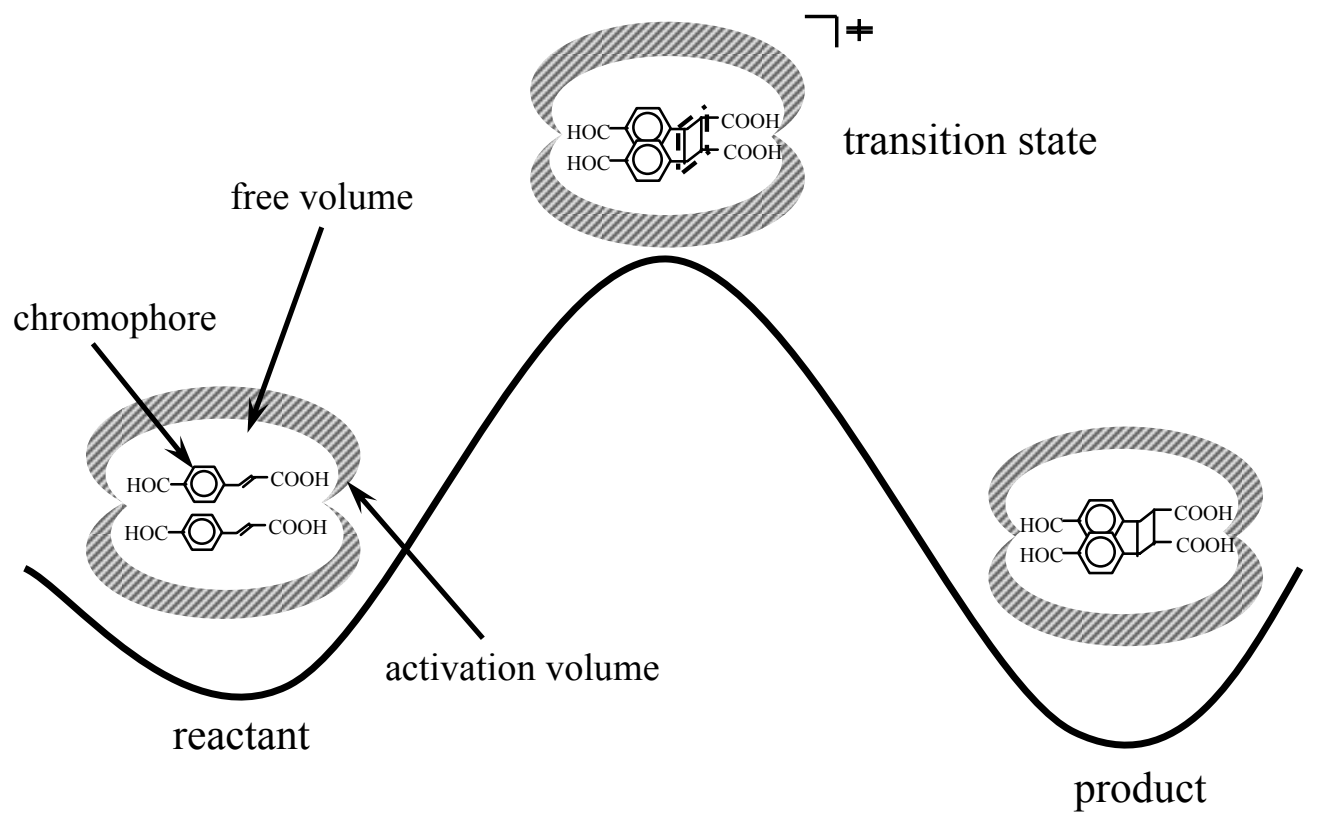

Figure 12: The definition of the reaction cavity and transition state of the [2+2] photodimerisation. 
The properties of the crystal package resulting from the thermodynamic crystallisation conditions have far-reaching consequences, e.g. for the application of such materials in optics or optoelectronics. Systems that follow the topochemical principle can be used as photodriven nanoabsorbers or materials for $3 \mathrm{D}$ holographic memory or - if the morphological change is reversible - as building blocks for photochromic materials [27].

For various reasons, the reaction mechanisms of topochemical reactions are still completely unknown: One reason for this can be found in the difficulty of measuring and interpreting the transient optical spectra due to the high optical density and the broad, structureless features in the spectra (Figure 13). Both, the reactant and the product do not show remarkable fluorescence in the UV range (according to our experiments, the fluorescence quantum yield is below $10^{-4}$ ) excluding also techniques based on fluorescence spectroscopy. Finally, topochemical reactions are of high interest for preparative chemists, because of their high stereoselectivity. Therefore, we have chosen the method of time-resolved x-ray structural analysis to study and elucidate in detail themechanism of this lightdriven solid state reaction.

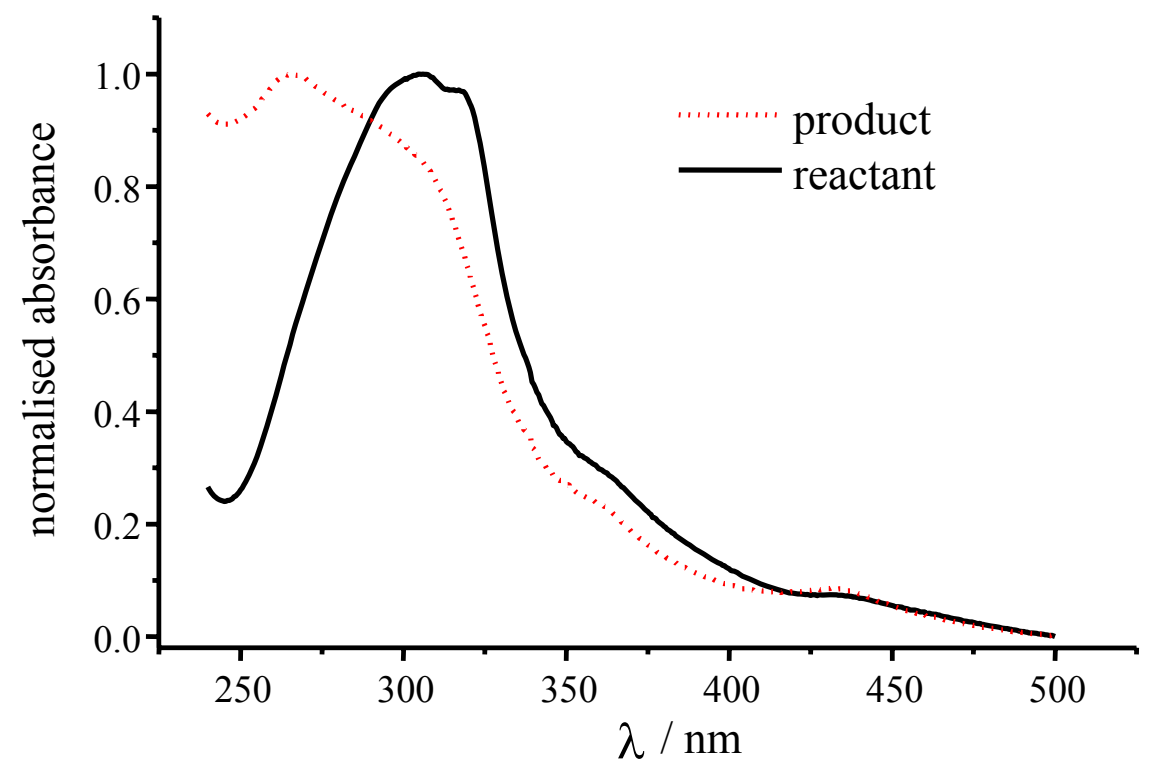

Figure 13: UV absorption spectrum of p-formyl-cinnamic acid: monomer (reactant) and dimer (product) spectra in the solid state. 
First we investigated the heterogeneous [2+2] photodimerisation reaction of $p$-formyltrans-cinnamic acid in powdereous materials (inset in Figure 11) [9]. When plotting the integrated intensity of the main scattering peak $C(t)$ as a function of the time delay between the optical pump and x-ray probe pulses, at very early times and within the time-resolution of the apparatus at ID09B at the ESRF, a fast modulation of the intensities of the Bragg diffraction peaks was observed. Figure 14 presents such a fast modulation for the most prominent Bragg diffraction peak. The lifetimes $\tau_{1}$ for the different samples represent the macroscopic overall transformation time of the sample to the amorphous product state for different sample thicknesses (for details see reference [9]).

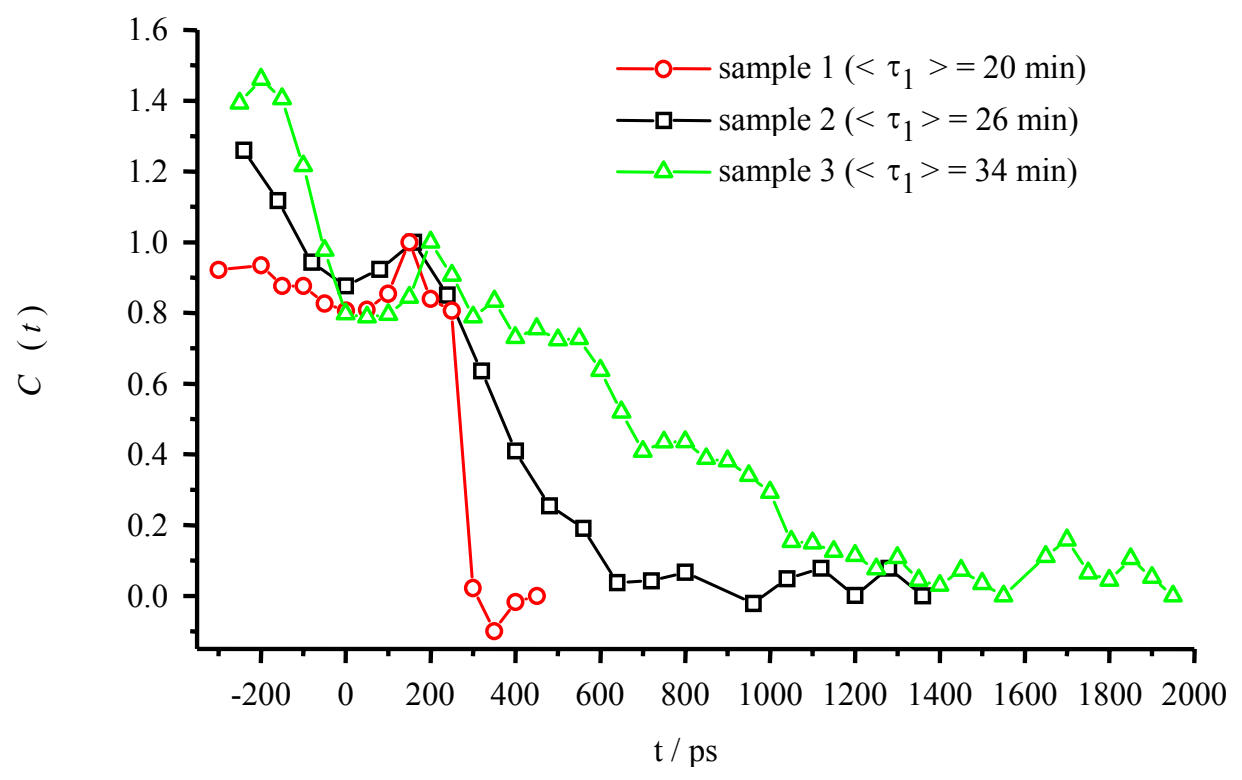

Figure 14: Fast time behaviour of the correlation function $C(t)$ of the most prominent Bragg diffraction peak for three representative samples. Note, that for clarity the correlation function is shown in a scaled way.

A possible mechanism for this reaction is proposed in Figure 15 including the formation of the fast intermediate state on the picosecond time scale and the overall transformation and amorphisation of the material on the minute time scale. 

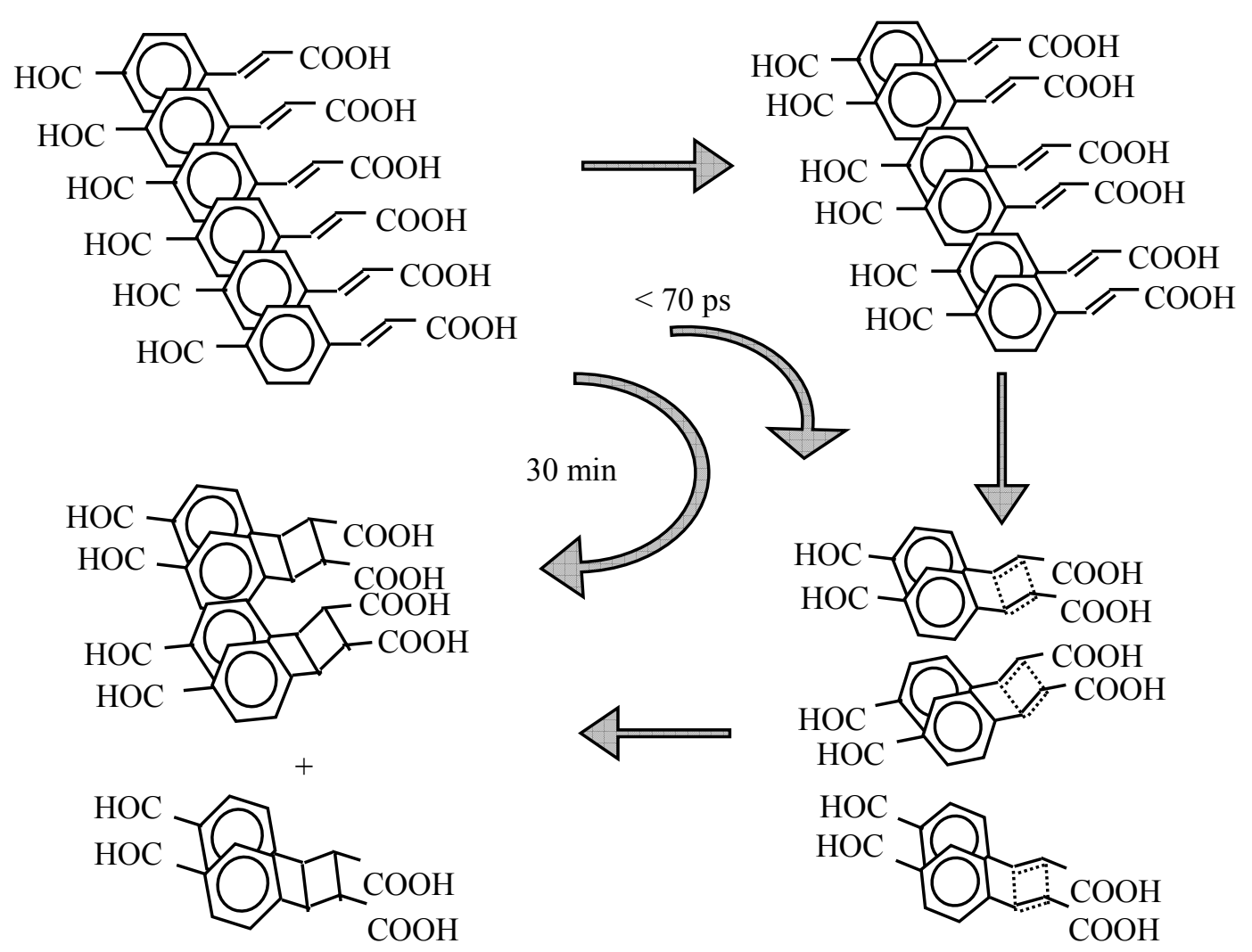

Figure 15: Suggested mechanism of the [2+2] photodimerisation of p-formyl-cinnamic acid.

In order to understand in more detail the possible mechanism of heterogeneous transformations, further investigations on cinnamic acid derivatives have been started [20,21]. Single crystals of 2,4-dichloro-trans-cinnamic acid $\left(\mathrm{C}_{9} \mathrm{H}_{6} \mathrm{Cl}_{2} \mathrm{O}_{2}\right.$, DiClCA) have been investigated using photocrystallography and optical spectroscopic methods (Figure 16).<smiles>O=C(O)/C=C/c1ccc(Cl)cc1Cl</smiles><smiles>CC1(C)C(C(=O)O)C(c2ccc(Cl)cc2Cl)C(C(=O)O)C1c1ccc(Cl)cc1Cl</smiles>

Figure 16: [2+2] photodimerisation of 2,4-dichloro-trans-cinnamic acid $\left(\mathrm{C}_{9} \mathrm{H}_{6} \mathrm{Cl}_{2} \mathrm{O}_{2}\right.$, DiClCA). 
The crystal was photo-excited on the red tail of the absorption spectrum of the DiClCA monomer with very gentle UV power $(<2 \mathrm{~mW})$ and no optical excess energy. Nonetheless, the kinetic analysis of the optical spectroscopic and the single crystal x-ray diffraction data of DiClCA upon UV excitation evidence a heterogeneous dimerization reaction (Figure 17, Figure 18).

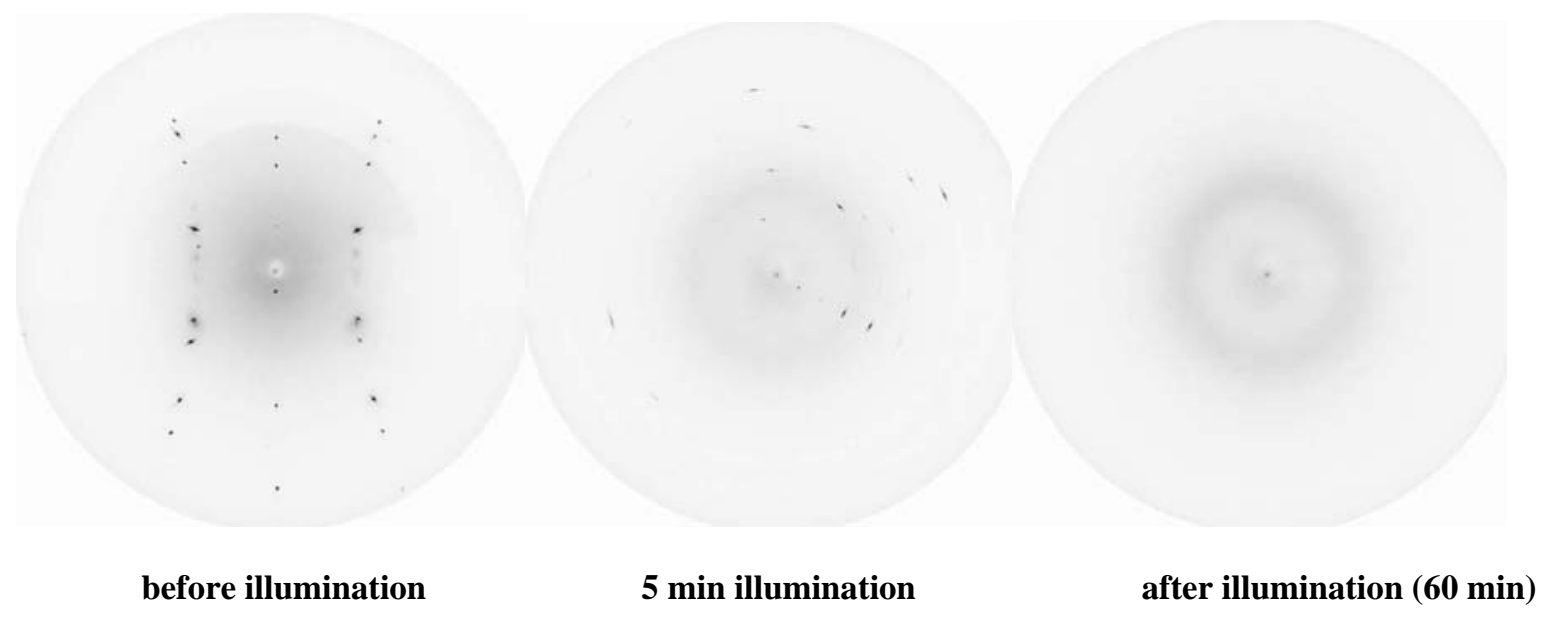

Figure 17: CCD snapshots of the DiClCA single crystal diffraction under illumination suggesting a heterogeneous product formation.

The time-scale of transformation of a typically $200 \times 200 \times 50 \mu \mathrm{m}^{3}$ crystal is around 12 minutes. Besides the bulk transformation, picosecond time-resolved experiments suggest similar kinetic features as been observed in p-formyl-trans-cinnamic acid [9]. 


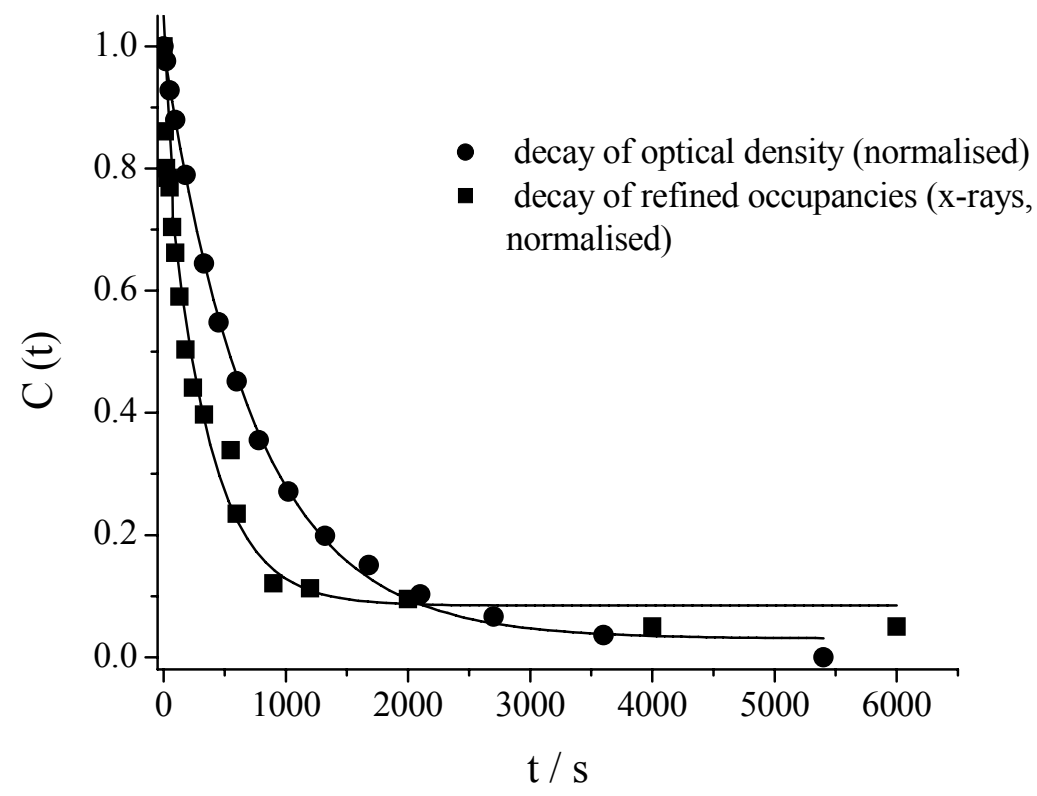

Figure 18: Comparison between the spectroscopic and the x-ray diffraction correlation functions of DiClCA photodimerization.

Taking into account the crystallographic results we suggest to include other dimensions of the monomer configurations in the crystal lattice [20]. In DiClCA the $\mathrm{C}=\mathrm{C}$ double bonds span a small angle of $5^{\circ}$ towards each other. In order to overcome this slight lack of parallelism during the reaction, the chromophores have to rearrange during the photo-reaction. Probably this rearrangement causes the formation of mosaic blocks and lead to a destruction of the long range order as the reaction progresses through the crystal.

Based on these experimental results a simplified kinetic reaction scheme for homogeneous and heterogeneous reactions has been proposed. In this scheme, the disordered dimer state has some kind of intermediate character for homogeneous reactions (Figure 19). The energy of the final ordered product state lowers as the reaction proceeds in the crystal. In heterogeneous reactions the activation energy cannot be gained out of the energy available in the system and the crystals transform to the amorphous state. 


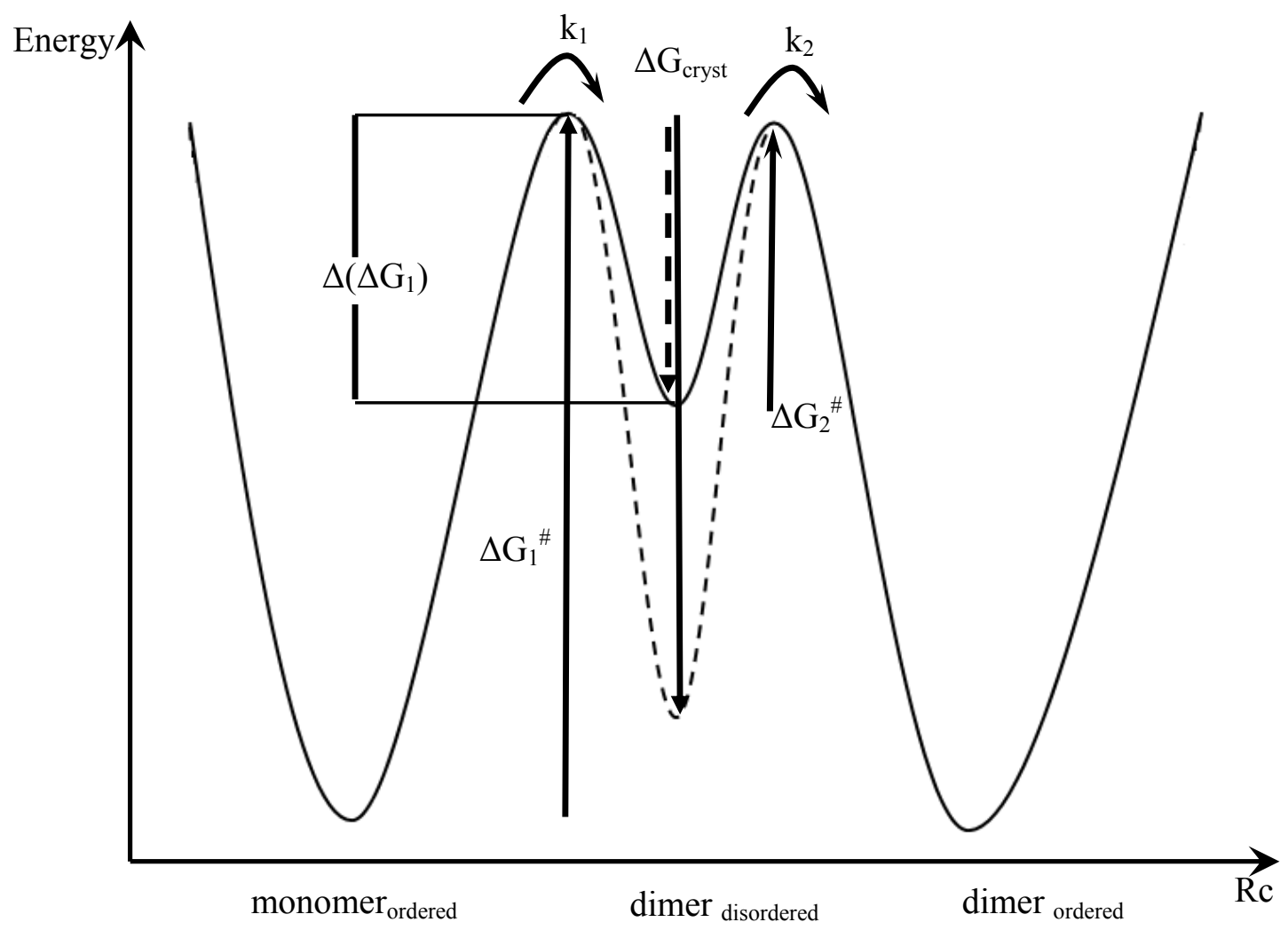

Figure 19: Reaction scheme of homogeneous and heterogeneous photodimerization reactions. In homogeneous reactions (solid curve, dashed arrow) the reaction progresses from an ordered monomer state to an ordered dimer state via the formation of a disordered dimer state as an intermediate or metastable state. The energy minimum of the dimer ${ }_{\text {ordered }}$ state lowers as the reaction proceeds in the crystal. In heterogeneous reactions the dimer ${ }_{\text {disordered }}$ state is stabilized in such a way, that the activation energy cannot be gained out of the energy available in the system. Now the disordered intermediate is a final product state and the crystals transform to this amorphous state (solid arrow, dashed curve). The population of the intermediate depends on the steepness of the dimer ${ }_{\text {disordered }}$ species inn the free energy diagram and hence on the ratio $k_{1} / k_{2}$ or $\Delta \mathrm{G}_{1}^{\#} /$ $\Delta \mathrm{G}_{2}{ }^{\#}$, respectively. Note, that depending on the system $\Delta\left(\Delta \mathrm{G}_{1}\right) \leq \Delta \mathrm{G}_{2}^{\#}$ or $\Delta\left(\Delta \mathrm{G}_{1}\right)>\Delta \mathrm{G}_{2}^{\#}$.

Also here we are currently studying more systems of interest in order to come to a more generlaised view of homo- and heterogeneous topochemical photodimerisation reactions [21]. 


\section{Outlook: Science with the X-ray Free Electron Laser}

The time-resolution of the reported time-resolved experiments is limited by the synchrotron x-ray pulse duration which is about $50-70$ ps. In order to achieve faster time domains the duration of the x-ray pulse has to be reduced. Here, future $\mathrm{x}$-ray free electron laser sources seem to be very attractive as they propose $\mathrm{x}$-ray pulse durations of the order of $100 \mathrm{fs}$ at photon fluxes even higher than present-day synchrotron radiation sources [28-30].

Employing the current detector technology, $10^{6} \mathrm{x}$-ray pulses with $10^{5}-10^{6}$ photons pulse $^{-1}$ have to be accumulated on the detector in order to obtain an x-ray signal of sufficient intensity. Using the X-FEL as source, few X-ray pulses would yield the same or more intensity at the detector. Therefore - due to the higher photon flux and the faster exposure times using the X-FEL, the investigations of irreversible reactions will also benefit from this source.

Currently, sub-ps resolution can be obtained at synchrotron sources if an IR-laser pulse triggered x-ray Streak camera is used as detection system. However, the low sensitivity of this device is not favourable for diffraction or diffuse scattering experiments with weak signal modulation on the femtosecond time sclae. Since x-ray pulses generated by XFELs have a natural pulsewidth of $100 \mathrm{fs}$, these sources will also offer better experimental conditions for the investigation of processes in the time domain from 100 fs to a few picoseconds, e.g. ultrafast structural relaxation experiments.

Recently, an international team could demonstrate that it is possible to couple an ultrafast optical laser to 100 fs long x-ray pulses, which were generated with a linear accelerator. The experiments were performed at the SubPicosecond Pulsed $x$-ray Source (SPPS at SSRL) generating typical $\mathrm{x}$-ray radiation of about $9 \mathrm{keV}$ [31,32]. With this setup the ultrafast structural changes in solids have been investigated [32]. For the demonstration experiments the semiconductor InSb was photoexcited to an intermediate state with quasiliquid character. This intermediate is formed within $100 \mathrm{fs}$ and has typical lifetimes of some 
picoseconds. It could be shown that the structural transition to the quasi-liquid phase is faster than 100 fs The experimental temporal time resolution was achieved by overcoming the laseraccelerator jitter which is on the order of picosecond by employing the method of the so-called electro-optical sampling to a time-resolution less than $100 \mathrm{fs}$ [31].

\section{Literature}

[1] J. R. Helliwell, P. M. Rentzepis, eds, Time-resolved Diffraction, Oxford Series on Synchrotron Radiation 2, Clarendon Press, Oxford (1997).

[2] a) V. Srajer, T.-Y. Teng, T. Ursby, C. Pradevard, Z. Ren, S. Adachi, W. Schildkamp, D. Bourgeois, M. Wulff, K. Moffat., Science 274, 1726 (1996).

b) F. Schotte, S. Techert, P. Anfinrud, V. Srajer, K. Moffat, M. Wulff, in Third-Generation Hard X-ray Synchrotron Radiation Sources, Ed. D. M. Mills, Wiley-Interscience, 345 (2001).

[3] S. Techert, F. Schotte, M. Wulff, Phys. Rev. Lett. 86 (10), 2030 (2001).

[4] S. Techert, J. Appl. Cryst. 37, 445 (2004).

[5] ] a) P. Coppens, I. Novozhilova, A. Kovalevsky, Chem. Rev. 102, 861 (2002).

b) M. R. Pressprich, M. A. White, Y. Vekhter, P. Coppens, J. Am. Chem. Soc., 1994, 116, 5233.

[6] M. Schmidt, R. Pahl, V. Srajer, S. Anderson, Z. Ren, H. Ihee, S. Rajagopal, K. Moffat, Proceed. Nat. Academ. Science 101 (14), 4799 (2004).

[7] J. Davaasambuu, P. Durand, S. Techert, J. Synchrotron Rad. 11, 483 (2004).

[8] a) R. Neutze, R. Wouts, S. Techert, A. Kirrander, J. Davidson, M. Kocsis, F. Schotte, M. Wulff, Phys. Rev. Lett. 87 (19), 195508 (2001).

b) A. Geis, D. Block, M. Burieau, F. Schotte, S. Techert, A. Plech, M. Wulff, H.P. Trommsdorff, J. of Luminesc. 94, 493 (2001). 
[9] G. Busse, Th. Tschentscher, A. Plech, M. Wulff, B. Frederichs, S. Techert, Faraday Discuss. 122, 105 (2003).

[10] E. Collet, M.-H. Lemée-Cailleau, M. Buron-Le Cointe, H. Cailleau, M. Wulff, T. Luty, S.-Y. Koshihara, M. Meyer, L. Toupet, P. Rabiller, S. Techert, Science 300, 612 (2003).

[11] C. Rischel, A. Rousse, I. Uschmann, P.-A. Albouy, J.-P. Geindre, P. Audebert, J.-C. Gauthier, E. Förster, J.-L. Martin, A. Antonetti, Nature 390, 490 (1997).

[12] C. Rose-Petruck, R. Jimenez, T. Guo, A. Cavalleri, C. W. Siders, F. Raksi, J. Squier, B. C. Walker, K. R. Wilson, C. P. J. Barty, Nature 398, 310 (1999).

[13] S. Techert, K. Zachariasse, J. Am. Chem. Soc. 126, 5593 (2004).

[14] L. X. Chen, W. J. H. Jägerm G. Jennings, D. J. Gosztola, A. Munkholm, J. P. Hessler, Science 292, 264 (2001).

[15] C. Bressler, M. Chergui, Chem. Rev.104, 1781 (2004).

[16] M. Klessinger and J. Michl, Lichtabsorption, Photochemie organischer Moleküle, VCH (1989).

[17] a) S. Techert, S. Schmatz, A. Wiessner und H. Staerk, Photophysical Characteristics of Directly Linked Pyrene-DMA Derivatives, J. Phys. Chem. A 104, 5700 (2000).

b) S. Techert, A. Wiessner, S. Schmatz and H. Staerk, J. Phys. Chem. B 105, 7579 (2001).

[18] a) S. Koshihara, Y. Tokura, K. Takeda and T. Koda, Phys. Rev. Lett. 68, 1148 (1992).

b) S. Koshihara, Y. Takahashi, H. Sakai, Y. Tokura, T. Luty, J. Phys. Chem. B 103, 2592 (1999).

[19] J. Davaasambuu, S. Techert, Light-induced Structural Variations in BBCP, J. Physics D: Appl. Physics, 38 (2005) A204-A207 (2005).

[20] J. Davasaambuu, G. Busse, S. Techert, J. Phys. Chem, in press (2005).

[21] A. Ramos, S. Techert, Biophys. J. 89, 1990-2003 (2005).

[22] M. D. Cohen, G. M. J. Schmidt, J. Chem. Soc. 1964, 1996 (1964).

[23] R. G. Weiss, V. Ramamurthy, G. S. Hammond, Acc. Chem. Res. 26, 530 (1993). 
[24] H. E. Zimmermann, E.E. Nesterov, Acc. Chem. Res. 2002 35, 77.

[25] F. Nakanishi, H. Nakanishi, T. Tasai, Y. Suzuki, M. Hasegawa, Chem. Lett. 525 (1974).

[26] D. E. Smith, C. B. Harris, J. Chem. Phys. 87, 2709 (1987).

[27] M. Irie, M., S. Kobatake, M. Horichi, Science 2001, 291, 176.

[28] a) G. Materlik, Th. Tschentscher (eds.), "Part V The X-ray Free Electron Laser," in: TESLA Technical Design Report, F. Richard et al. (eds.), TESLA-FEL 2001-05, DESY, Hamburg (March 2001).

b) S. Techert and R. Neutze, "Ultrafast Chemistry I" eds. G. Materlik, Th. Tschentscher (eds.) in: TESLA Technical Design Report,Part V, F. Richard et al. (eds.), TESLA-FEL 2001-05, DESY, Hamburg (March 2001).

c) S. Techert, "Ultrafast Chemistry II", Th. Tschentscher (ed.) in: TESLA Technical Design Report (Progress Report), F. Richard et al. (eds.), TESLA-FEL 2001-05, DESY, Hamburg (March 2001).

[29] Th. Tschentscher, Proceedings of SPIE 4500, 1 (2001).

[30] C. Blome, Th. Tschentscher, J. Davaasambuu, P. Durand and S. Techert, J. Synchrotron Rad. 12, 812-819 (2005).

[31]A. L. Cavalieri, D. M. Fritz, S. H. Lee, P. H. Bucksbaum, D. A. Reis, J. Rudati, D. M. Mills, P. H. Fuoss, G. B. Stephenson, C. C. Kao, D. P. Siddons, D. P. Lowney, A. G. MacPhee, D. Weinstein, R. W. Falcone, R. Pahl, J. Als-Nielsen, C. Blome, S. Düsterer, R. Ischebeck, H. Schlarb, H. Schulte-Schrepping, Th. Tschentscher, J. Schneider, O. Hignette, F. Sette, K. Sokolowski-Tinten, H. N. Chapman, R. W. Lee, T. N. Hansen, O. Synnergren, J. Larsson, S. Techert, J. Sheppard, J. S. Wark, M. Bergh, C. Caleman, G. Huldt, D. van der Spoel, N. Timneanu, J. Hajdu, R. A. Akre, E. Bong, P. Emma, P. Krejcik, J. Arthur, S. Brennan, K. J. Gaffney, A. M. Lindenberg, K. Luening, J. B. Hastings, Phys. Rev. Lett. 94, 114801 (2005). 
[32] A. M. Lindenberg, J. Larsson, K. Sokolowski-Tinten, K. Gaffney, C. Blome, O. Synnergren, J. Sheppard, C. Caleman, A. G. MacPhee, D. Weinstein, D. P. Lowney, T. Allison, T. Matthews, R. W. Falcone, A. L. Cavalieri, D. M. Fritz, S. H. Lee, P. H. Bucksbaum, D. A. Reis, J. Rudati, D. M. Mills, P. H. Fuoss, G. B. Stephenson, C. C. Kao, D. P. Siddons, R. Pahl, J. Als-Nielsen, S. Dusterer, R. Ischebeck, H. Schlarb, H. ShulteSchrepping, Th. Tschentscher, J. Schneider, O. Hignette, F. Sette, H. N. Chapman, R. W. Lee, T. N. Hansen, S. Techert, J. S. Wark, M. Bergh, G. Huldt, D. van der Spoel, M. Timneanu, J. Hajdu, D. von der Linde, R. A. Akre, E. Bong, P. Emma, P. Krejcik, J. Arthur, S. Brennan, K. Luening, J. B. Hastings, Science 308, 392-394 (2005). 